\title{
Leguminosas da Amazônia Brasileira - XV. O pólen do gênero Bocoa Aubl. (Leguminosae - Papilionoideae)
}

\author{
Ely Simone Cajueiro Gurgel ${ }^{1}$, Léa Maria Medeiros Carreira ${ }^{2}$ e Maura Anjos de Andrade Kalume ${ }^{1}$
}

Recebido em 06/04/2002. Aceito em 19/11/2003

\begin{abstract}
RESUMO - (Leguminosas da Amazônia Brasileira - XV. O pólen do gênero Bocoa Aubl. (Leguminosae - Papilionoideae)). O gênero Bocoa Aubl. (Leguminosae - Papilionoideae), está representado na Amazônia brasileira pelas espécies Bocoa alterna (Benth.) Cowan, B. racemulosa (Huber) Cowan e B. viridiflora (Ducke) Cowan. Botões florais adultos foram coletados de amostras depositadas nos herbários MG e INPA, e acetolisados para posterior preparo de lâminas. Com o objetivo de analisar a morfologia polínica, os grãos de pólen foram medidos, descritos e fotomicrografados. Os grãos de pólen das espécies estudadas são 3-colporados, variam de pequenos a médios, de subprolatos a prolatos, o amb de subtriangular a triangular e a superfície de punctada a microrreticulada. Os grãos de pólen das espécies analisadas são homogêneos, tratando-se, portanto, de um gênero estenopalino. Foi elaborada uma chave de identificação, com base nos caracteres polínicos, a fim de separar as espécies.
\end{abstract}

Palavras-chave: morfologia polínica, Leguminosae - Papilionoideae, Amazônia brasileira, Bocoa

\begin{abstract}
Brazilian Amazon Legumes - XV. Pollen of the genus Bocoa Aubl. (Leguminosae-Papilionoideae)). The genus Bocoa Aubl. (Leguminosae - Papilionoideae) is represented in Brazilian Amazon for species Bocoa alterna (Benth.) Cowan, B. racemulosa (Huber) Cowan and B. viridiflora (Ducke) Cowan. With the objetive to analyse the pollinic morphology, adults flower-buds were colleted to the MG and INPA herbarium, and submited to acetolisis and slides preparations. The pollens grains were measured, described, and photomicrographed. The pollen grains are 3-colporate, vary from small to medium size, subprolate to prolate, amb subtriangular to triangular, and surface punctate to microreticulate. The pollen grains of the species analysed were homogeneous, so it is a stenopalynous genus. A key based in polinic caracters was elaborate to separate the species.
\end{abstract}

Key words: pollen morphology, Leguminosae - Papilionoideae, Brazilian Amazon, Bocoa

\section{Introdução}

Bocoa Aubl. faz parte da família Leguminosae, subfamília Papilionoideae (Cowan 1981) e segundo Silva et al. (1989), encontra-se representado na Amazônia brasileira pelas espécies Bocoa alterna (Benth.) Cowan, Bocoa racemulosa (Huber) Cowan e Bocoa viridiflora (Ducke) Cowan, esta tratando-se de uma árvore cujo porte varia de mediano a grande e vulgarmente conhecida como "muirajibóia-preta" e "muirajibóia - amarela" (Silva et al. 1977; 2002).

A posição taxonômica do gênero na tribo Swartzieae tem sido motivo de discussão entre diversos autores. Ducke (1922) fez breve descrição da espécie Bocoa racemulosa, delimitando sua zona de ocorrência, que vai dos rios Trombetas ao Tapajós, no Estado do Pará. Lewis (1987) fez descrições taxonômicas das espécies Bocoa limae Cowan e B. mollis (Benth.) Cowan, mais sucintas ainda que as de Ducke (1922).
Em relação a estudos já realizados sobre a morfologia polínica, Cowan (1974) analisou os grãos de pólen de espécies dos gêneros Swartzia Schreb., Bocoa Aubl., Andira Juss., Zollernia Wied-Neuw. \& Nees, Lecointea Ducke, Candolleodendron R.S. Cowan e Holocalyx Micheli, separando-os em tipos polínicos. Carreira et al. (1996) descreveram os grãos de pólen de Bocoa alterna (Benth.) Cowan.

Este estudo tem como principal objetivo analisar os grãos de pólen das espécies de Bocoa que ocorrem na Amazônia brasileira, a fim de obter resultados que possam contribuir para futuras pesquisas relacionadas a este gênero.

\section{Material e métodos}

Botões florais adultos foram retirados de amostras depositadas nos herbários: MG (Museu Paraense Emílio Goeldi, Belém, PA) e INPA (Instituto Nacional

\footnotetext{
Bolsistas de Iniciação Científica do PIBIC/CNPq/MPEG/DBO, Processo 100508/94-4

2 MCT/CNPq, Museu Paraense Emílio Goeldi (MPEG), DBO, C. Postal 399, CEP 66040-170, Belém, PA, Brasil
} 
de Pesquisas da Amazônia, Manaus, AM) e posteriormente submetidos à acetólise (Erdtman 1952). A relação das espécies estudadas, com suas respectivas referências de herbário e de palinoteca, encontra-se na Tabela 1.

Após a preparação das lâminas foram procedidas as medidas em microscópio ZEISS adaptado com escala micrometrada. As dos eixos polar e equatorial, em 25 grãos de pólen, em vista equatorial. Com estes valores, foram calculados a média, o desvio padrão e o coeficiente de variação. Para as medidas da sexina, nexina, do diâmetro do lúmen e da endoabertura, em 10 grãos de pólen, foi calculada somente a média aritmética.

Para as observações em MEV, os grãos de pólen, após a acetólise, foram desidratados e permaneceram por 24 horas em acetona a $50 \%$ e, posteriormente, em acetona a $100 \%$ durante 30 minutos. Uma gota da suspensão de pólen em acetona pura foi depositada sobre o suporte do MEV por algumas horas a $37^{\circ} \mathrm{C}$, antes de ser evaporada com ouro.

As informações sobre a ornamentação da exina nas descrições polínicas foram feitas baseadas nas fotomicrografias em MEV.

Nas descrições polínicas foram usadas a sequiência padronizada de Erdtman (1969) e a classificação de Praglowski \& Punt (1973), sendo que a nomenclatura foi baseada em Barth \& Melhem (1988) e Punt et al. (1998).

As fotomicrografias de luz foram obtidas em um fotomicroscópio ZEISS e as de MEV, em microscópio eletrônico de varredura ZEISS (modelo DSM-940). As fotomicrografias em ML de B. viridiflora não foram obtidas devido à falta de equipamento óptico.

Nas descrições e nas legendas das figuras e da tabela foram usadas as abreviaturas: amb - âmbito; cp - colpo; E - eixo equatorial; end - endoabertura; MEV - microscopia eletrônica de varredura; ML - microscopia de luz; Nex - nexina; NPC - número, posição e caráter das aberturas; $\mathrm{P}$ - eixo polar; $\mathrm{P} / \mathrm{E}$ - relação entre as medidas dos eixos polar e equatorial; P/MG - número da Palinoteca do Museu Paraense Emílio Goeldi; s.d. - sem data; Sex - sexina; s.n. - sem número; VE - vista equatorial do grão de pólen e VP - vista polar do grão de pólen.

\section{Resultados}

Caracteres gerais dos grãos de pólen do gênero - Grãos de pólen isopolares, de simetria radial, 3-colporados, $\mathrm{NPC}=345$. Variam de pequenos a médios, o $a m b$ de subtriangular a circular, a forma de prolata esferoidal a subprolata, a superfície de punctada a microrreticulada, a endoabertura de circular a lolongada. A espessura da sexina é igual ou quase igual à da nexina.

Descrições polínicas das espécies - 1) B. alterna (Benth.) Cowan (Fig. $1 \mathrm{a}-\mathrm{g}$ ): $a m b$ subtriangular, forma subprolata e de superfície punctada. A endoabertura é lolongada $(4,82 \times 2,73 \mu \mathrm{m}) . \mathrm{P}=27,0 \pm 0,7(26,0-31,0) \mu \mathrm{m}$; $\mathrm{E}=22,0 \pm 0,7(20,0-25,0) \mu \mathrm{m} ; \mathrm{P} / \mathrm{E}=1,21 . \mathrm{Sex}=0,7 \mu \mathrm{m}$; Nex $=0,6 \mu \mathrm{m} .2$ ) B. racemulosa (Huber) Cowan (Fig. 2 a-g): amb triangular, forma subprolata e de superfície punctada. A endoabertura é circular $(4,13 \mu \mathrm{m}) . \mathrm{P}=30,0 \pm 0,6(29,0-33,0) \mu \mathrm{m} ; \mathrm{E}=25,0 \pm 0,6$ $(22,0-26,0) \mu \mathrm{m} ; \mathrm{P} / \mathrm{E}=1,22 . \mathrm{Sex}=1,0 \mu \mathrm{m} ; \mathrm{Nex}=0,6 \mu \mathrm{m}$. 3) B. viridiflora (Ducke) Cowan (Figura 3 a - c): $a m b$ triangular, forma prolata e de superfície microrreticulada. A endoabertura é circular $(4,60 \mu \mathrm{m})$. $\mathrm{P}=18,0 \pm 0,6(17,0-22,0) \mu \mathrm{m} ; \mathrm{E}=13,0 \pm 0,9$ $(11,0-19,0) \mu \mathrm{m} ; \mathrm{P} / \mathrm{E}=1,36 ; \mathrm{DL}=0,72 \mu \mathrm{m}$. Sex $=0,7 \mu \mathrm{m}$; $\mathrm{Nex}=0,4 \mu \mathrm{m}$.

\section{Chave polínica}

1. Grãos de pólen de superfície microrreticulada, com endoabertura circular Bocoa viridiflora

1. Grãos de pólen de superfície punctada

2. Endoabertura lolongada Bocoa alterna

2. Endoabertura circular Bocoa racemulosa

Tabela 1. Relação das espécies de Bocoa Aubl. estudadas com suas respectivas referências de herbário e de palinoteca.

\begin{tabular}{|c|c|c|c|c|c|c|}
\hline Espécie & Coletor & Data de coleta & N. herbário & N. palinoteca & Nomes vulgares & Procedência \\
\hline B. alterna (Benth.) Cowan & L. Coelho s.n. & s.d. & MG 24950 & P/MG-01135 & gombeira & Amazonas \\
\hline B. racemulosa (Huber) Cowan & E. Snethlage s.n. & $04 / 11 / 1908$ & MG 10058 & P/MG-01136 & - & Pará \\
\hline B. viridiflora (Ducke) Cowan & A. Loureiro s.n. & $26 / 10 / 1965$ & INPA 16456 & P/MG-01343 & $\begin{array}{l}\text { muirajibóia-amarela; } \\
\text { muirajibóia-preta }\end{array}$ & Amazonas \\
\hline
\end{tabular}



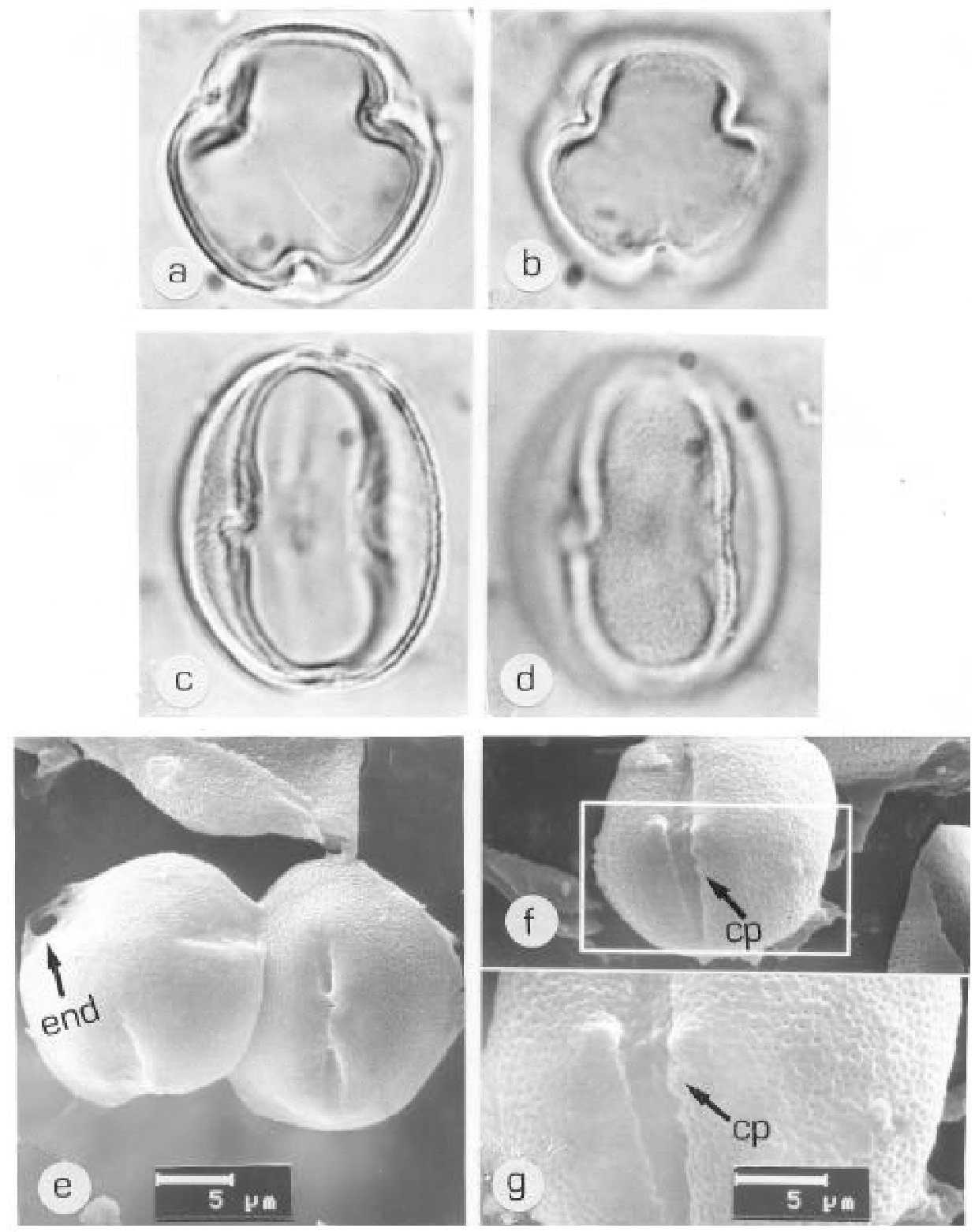

Figura 1. Pólen de Bocoa alterna (Benth) Cowan. ML: A. VP, corte óptico. B. Idem, aspecto da ornamentação da exina. C. VE, corte óptico. D. Idem, aspecto da ornamentação da exina (1600x). MEV: E. VP e VE, aspecto dos colpos e endoabertura. F. VE, aspecto do colpo. G. Detalhes da ornamentação da exina e do colpo.

\section{Discussão}

Cowan (1974) analisou o pólen de algumas espécies dos gêneros que constituem a tribo Swartzieae e, baseado no comprimento dos colpos e padrão da exina, estabeleceu cinco tipos polínicos, sendo que as espécies $B$. alterna e $B$. racemulosa, aqui estudadas, enquadram-se no grupo 1, Tipo I-A.

Em seus estudos, Cowan (1974) chegou à conclusão de que os grãos de pólen das espécies investigadas são muito semelhantes e para distingui-los seria necessário utilizar técnicas mais sofisticadas. Por esta razão, o autor ficou convencido que os grãos de pólen deltóide-globosos ou deltóides não ocorrem no gênero, acrescentando que os grãos de pólen das espécies examinadas são mônades, eixo polar com cerca de $18-30 \mu \mathrm{m}$ compr., esferoidais, subprolatos ou prolatos, 3-colporados, com colpo curto, alongado ou sincolpado. A endoabertura é circular, freqüentemente proeminente, algumas vezes encoberta pela sexina, que é mais ou menos psilada, punctada, fina ou grosseiramente rugosa, ou estriado-reticulada. 
Os grãos de pólen de B. alterna foram descritos por Carreira et al. (1996) como psilados. À despeito de ter sido analisada amostra diferente, os grãos de pólen aqui analisados foram descritos como punctados, não concordando, portanto, com os referidos autores.

As características polínicas das espécies aqui analisadas permitiram considerar que Bocoa é estenopolínico, uma vez que a homogeneidade entre seus grãos de pólen é bastante significativa, principalmente quanto ao número de aberturas e ornamentação da exina. Os tipos de superfície, microrreticulada e punctada, empregados para caracterizar os grãos de pólen das espécies aqui investigadas, correspondem ao tipo rugoso descrito por Cowan (l.c.), à despeito de terem sido usadas nomenclaturas mais atualizadas para descrevê-los, como a de Praglowski \& Punt (1973), e a de Punt et al. (1998).

\section{Agradecimentos}

À Dra. O.M. Barth, pelas fotomicrografias obtidas no MEV do Instituto Oswaldo Cruz.
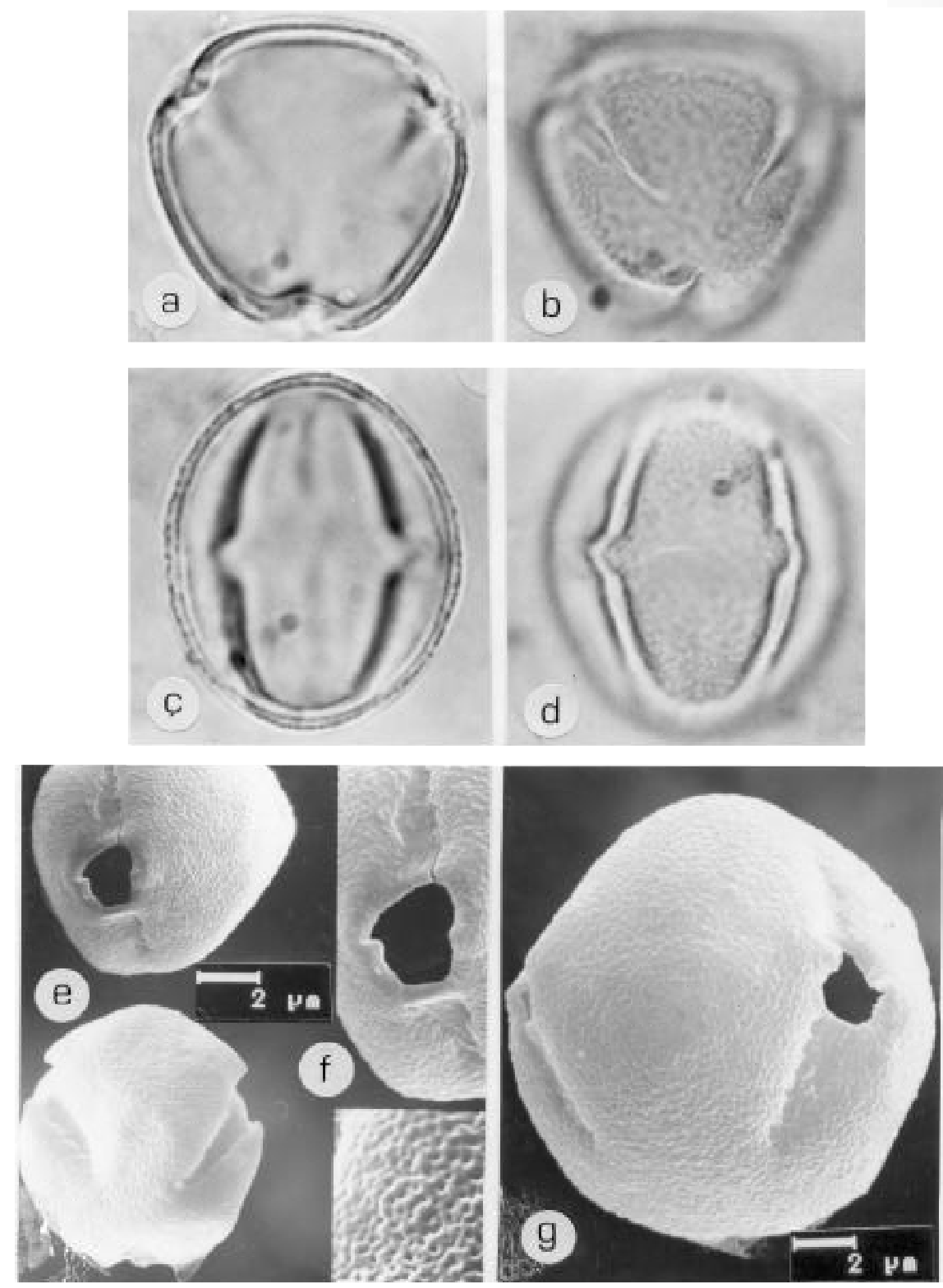

Figura 2. Pólen de Bocoa racemulosa (Huber) Cowan. ML: A. VP, corte óptico; B. Idem, aspecto da ornamentação da exina; C. VE, corte óptico; D. Idem, aspecto da ornamentação da exina (1600x). MEV: E. VE e VP, aspecto dos colpos e endoabertura; F. VE, aspecto dos colpos e endoabertura; G. VE, detalhes da endoabertura e da ornamentação da exina. 

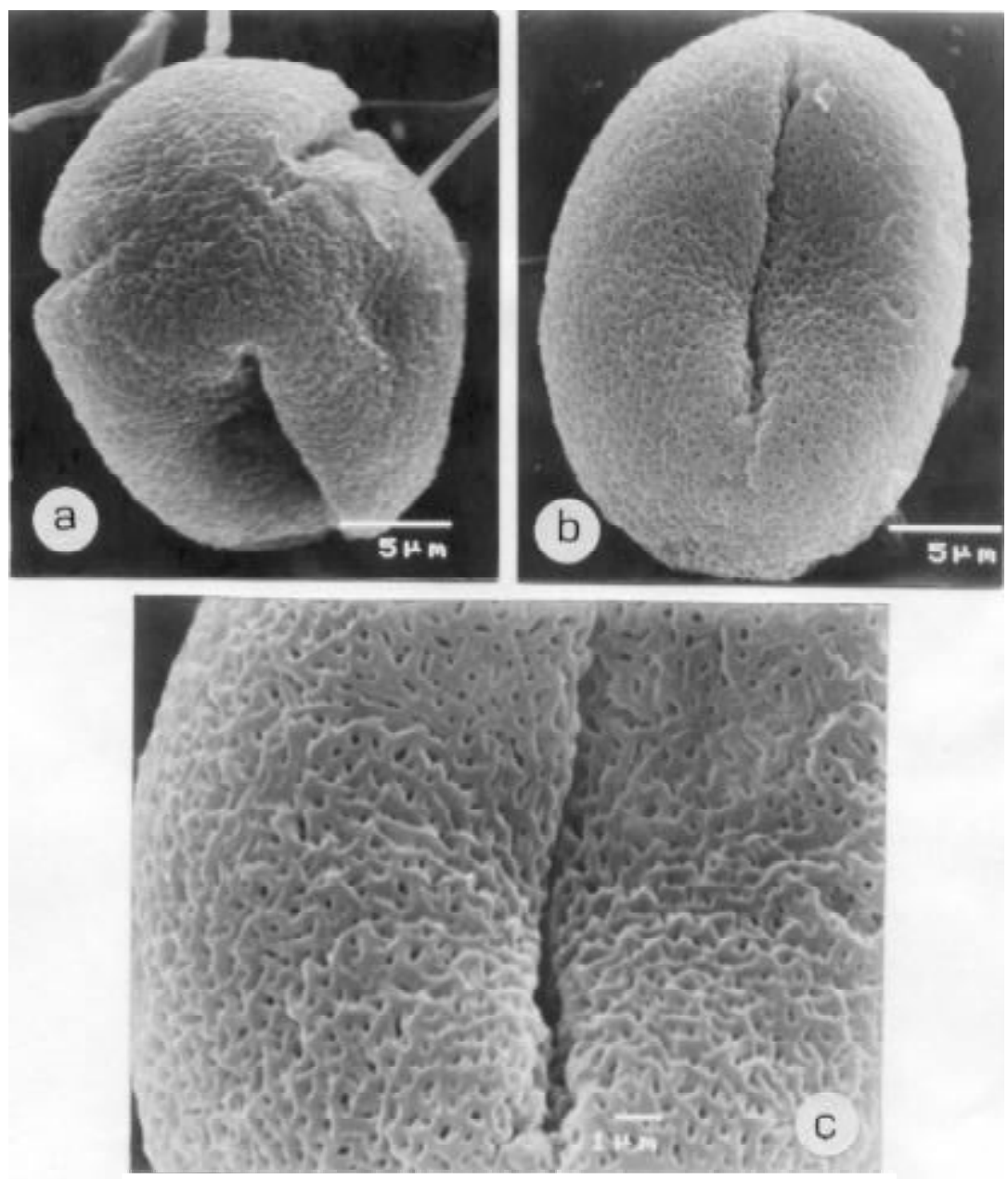

Figura 3. Pólen de Bocoa viridiflora (Ducke) Cowan. MEV: A. VP, evidenciando a extremidade dos colpos; B. VE, aspecto do colpo; C. Detalhes do colpo e da ornamentação da exina.

\section{Referências bibliográficas}

Barth, O. M. \& Melhem, T. S. 1988. Glossário Ilustrado de Palinologia. Unicamp, Campinas.

Carreira, L.M.M.; Lopes, J.R.C.; Silva, M.F. \& Nascimento, L.A.S. 1996. Catálogo de Pólen das Leguminosas da Amazônia Brasileira. Museu Paraense Emílio Goeldi, Coleção Adolpho Ducke, Belém. 137p.: il.

Cowan, R.S. 1974. A revison of the genus Bocoa. (Leguminosae Papilionoideae). Proceedings of the Biological Society of Washington 87(13): 95-127.

Cowan, R.S. 1981. Swartzieae. In: R.M. Polhill \& P.H. Raven (eds.). Advances in Legume Systematic 1: 209-212.

Ducke, A. 1922. Plantes Nouvelles ou peu connues de la région Amazoniénne. II. Arquivos do Jardim Botânico do Rio de Janeiro 3: 47-175.

Erdtman, G. 1952. Pollen Morphology and plant TaxonomyAngiosperms. Almquist \& Wiksell, Stockholm.
Erdtman, G. 1969. Handbook of Palinology. Hafner, New York. Lewis, G.P. 1987. Legumes of Bahia. Royal Botanic Gardens, Kew, Richmond, Surrey.

Punt, W.; Blackmore, S.; Nilsson, S. \& Le Thomas, A. 1998. Glossary of pollen and spores terminology. 2 ed. LPP Foundation, Utrecht.

Praglowski, J. \& Punt, W. 1973. An elucidation of the microreticulate structure of the exine. Grana 13: 45-50.

Silva, M.F.; Lisboa, P.L.B. \& Lisboa, R.C.L. 1977. Nomes vulgares de plantas amazônicas. INPA, Belém.

Silva, M. F.; Carreira, L.M.M.; Tavares, A.L.; Ribeiro, I.C.; Jardim, M.A.G.; Lobo, M.G.A. \& Oliveira, J. 1989. As Leguminosas da Amazônia Brasileira - Lista Prévia. Acta Botanica Brasílica 2(suplemento): 193-237.

Silva, M.F.; Souza, L.A. \& Carreira, L.M.M. 2004. Nomes populares das Leguminosas do Brasil. Editora da Universidade Federal do Amazonas, Manaus. 236p. 